\title{
Search for Oscillations with a Lithium-6 Detector at the SCK•CEN BR2 reactor
}

\author{
Simon Vercaemer ${ }^{*}$ \\ Vrije Universiteit Brussel, \\ Universiteit Antwerpen \\ E-mail: simon.vercaemer@vub.be
}

As submitted:

Several anomalies in the neutrino sector are pointing towards the existence of a new (sterile) neutrino state with a mass around $1 \mathrm{eV}$. The SoLid experiment is located at the SCK•CEN BR2 research reactor in Belgium and will investigate this possibility. Using the large flux of anti-neutrino generated in the reactor, it will collect a high statistics sample of Inverse Beta Decay (IBD) events. These will be used to study the energy and distance dependence of the neutrino flux, which in turn will be used to unambiguously support or reject the evidence of sterile neutrinos being the cause of these anomalies.

The measurement is challenging as one has to operate a detector very close to the high radiation environment of a nuclear reactor and on the surface with little overburden to shield against cosmic rays. SoLid is employing a new technology using highly segmented scintillators with excellent particle ID to face these challenges.

The 1.6-tons detector was installed towards the end of 2017 and is taking data since early 2018. We will describe the detector design, the experimental setup at BR2 and the detection principle. This will be followed by a first look at the data.

The 39th International Conference on High Energy Physics (ICHEP2018)

4-11 July, 2018

Seoul, Korea

\footnotetext{
${ }^{*}$ Speaker.

${ }^{\dagger}$ for the SoLid collaboration
} 


\section{The SoLid experiment: motivation, location and working principles}

Observation of the antineutrino rate coming from nuclear reactors has resulted in a rate lower than expected. This deficit, the so-called Reactor Anti-neutrino Anomaly (RAA), has been observed by multiple collaborations and over a wide range of distances between the detector and the reactor core $[1,2]$. Combined with other observed deviations, gallium and LSND/MiniBooNE, the anomalies reach a large significance. All three anomalies could be resolved by the introduction of a fourth 'sterile' neutrino with a squared mass splitting of the order $1 \mathrm{eV}^{2}$ and an appropriately small mixing angle. Since the number of active neutrinos has been determined to three, this new neutrino can not interact with ordinary matter and is therefore 'sterile'. The characteristics of the sterile neutrino can be determined from the disappearance pattern imposed on the distance and energy spectrum of neutrino interactions. To this end, the SoLid collaboration designed and built a voxelized hybrid scintillator antineutrino detector, a completely new design. The detector is constructed out of 12,800 detection cells arranged in 50 planes of 16 by 16 cells, split in five modules of ten planes each. Every single detection cell consists of a $5 \times 5 \times 5 \mathrm{~cm}^{3}$ PVT cube and two ${ }^{6} \mathrm{LiF}: \mathrm{ZnS}(\mathrm{Ag})$ screens wrapped in Tyvek for optical insulation. The PVT cubes act as a neutrino target, providing the protons required for the antineutrino to undergo the IBD reaction: $\bar{v}_{e}+{ }_{1}^{1} p \rightarrow e^{+}+{ }_{1}^{0} n$ and as scintillator for the positron, from which both the location and the energy of the neutrino interaction can be determined.

Due to the backgrounds involved with operating a neutrino detector on the Earth's surface and next to a nuclear reactor, the coincidence of the positron with the neutron is required. The neutron thermalizes via elastic scattering on the detector materials and is then captured on a ${ }^{6} \mathrm{Li}$ nucleus. This capture reaction produces an alpha and a tritium nucleus, sharing $4.78 \mathrm{MeV}$ of kinetic energy. Both are highly ionizing and deposit all their energy within the ${ }^{6} \mathrm{LiF}: \mathrm{ZnS}(\mathrm{Ag})$ screen, scintillating in the $\mathrm{ZnS}(\mathrm{Ag})$ microcrystals. The thermalization process leads to a distribution in the time and distance between the positron and the neutron signals, this is shown in Figure 1.
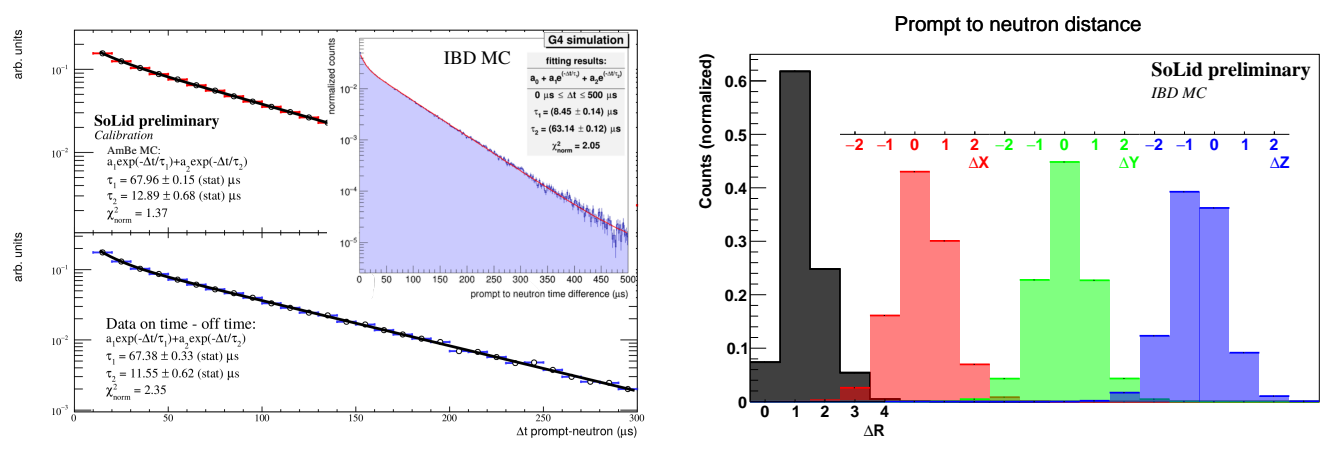

Figure 1: The time (inset left) and spatial (right) coincidence distributions of simulated IBD events. For the time distribution, a comparison using an AmBe neutron source is included, showing the time distribution for both simulation (top left) and data (bottom left). 
Light is extracted from the detection cells via an orthogonal grid of wavelength shifting (WLS) fibres threaded in groves cut in the sides of the PVT cubes. Four WLS fibres cross each cube, two in the $X$ and two in the $Y$ direction. The coincidence between the $X$ and $Y$ fibres allows determining the cube where the light originated. The WLS fibres are read out by SiPMs on one side and have a mirror on the other side, the two WLS fibres per direction guarantee a homogeneous readout by placing a SiPM at each end of a row or column of cubes [3].

Light coming from PVT and $\mathrm{ZnS}(\mathrm{Ag})$ scintillation is collected through the same WLS fibres, the separation is made using the different time constants of the two scintillators. PVT has a very short time constant (2.7 ns) while $\mathrm{ZnS}(\mathrm{Ag})$ has a much longer time constant $(80 \mu \mathrm{s})[4,5]$. An energy deposition in respectively PVT and ZnS(Ag) therefore leads to a single sharp peak and a long signal with a multitude of peaks. A dedicated neutron trigger was developed in order to select $\mathrm{ZnS}(\mathrm{Ag})$ scintillations based on the number of peaks detected in a time window [6].

The SoLid experiment monitors SCK•CEN's BR2 reactor in Mol, Belgium. This research reactor is highly suited for an ultra short baseline oscillation measurement. Thanks to it's compact core $(\varnothing \approx 0.5 \mathrm{~m})$, very little position smearing takes place. The smallness of the core also allows a close approach, as little as $5.5 \mathrm{~m}$. No interfering experiments currently take place in the experimental hall and all beam ports have been closed, leading to a favourable environment compared not only to commercial reactors but also to other research reactors. The month long reactor cycles with intermittent reactor off periods allow for an accurate background determination. This reactor schedule results in a duty cycle of roughly $40 \%$ with a power of $60 \mathrm{MW}_{\text {therm }}$ when the reactor is on.

\section{Backgrounds}

Apart from accidental coincidences, a number of correlated backgrounds are present. Due to the low overburden $(\sim 10$ m.w.e.), spallation neutrons from cosmic ray muon interactions and atmospheric neutrons produced in the initial cosmic ray interactions, i.e. the cosmic backgrounds, are a relevant background. Spallation neutrons are tackled via a muon veto. Neutrons produced in spallation reaction have the same thermalization and capture time shown in Figure 1. A muon veto of $200 \mu$ s reduces this background by $95 \%$ while inducing a $6 \%$ dead time. In atmospheric neutron events, on average 1.7 neutrons are captured. A neutron multiplicity veto identical to the muon veto reduces the high multiplicity component of this background by $94.5 \%$. The single atmospheric neutrons are rejected using selection criteria such as energy, prompt multiplicity and the spatial distributions shown in Figure 1.

Another correlated background is due to the natural radioactivity of the detector materials and its surroundings. In the uranium decay chain, decay of ${ }^{214} \mathrm{Bi}$ to ${ }^{214} \mathrm{Po}$ to ${ }^{212} \mathrm{~Pb}$ mimics the IBD process. ${ }^{214} \mathrm{Bi}$ decays via $\beta^{-}$decay with a $Q$-value of $3.2 \mathrm{MeV}$, emitting an electron in the PVT. The subsequent $\alpha$ decay of ${ }^{214}$ Po has a half time of $164 \mu \mathrm{s}$, close to the characteristic neutron capture time of $63 \mu \mathrm{s}$. When the $\alpha$ is emitted into the $\mathrm{ZnS}(\mathrm{Ag})$, it generates a signal very similar to that expected from neutron capture. The so-called 
BiPo background is reduced via the same topological selections that are also used to reduce single atmospheric neutrons. Investigations into discriminating the $\mathrm{ZnS}(\mathrm{Ag})$ signal produced by an $\alpha$ particle from the signal generated by neutron capture are ongoing.

\section{Detector performance}

The SoLid detector has been taking data since February 2018 with commissioning data available for December 2017 as well. In the commissioning data, part of the shielding was not yet constructed and only four of the five modules were installed. For a first look at the data, the data collected during the commissioning period and in May 2018 were made available. Both periods have reactor on and reactor off data, albeit more reactor on than reactor off. In both datasets, a clear excess of IBD-like events during the reactor on periods is observed. The rate per day of IBD like events using a preliminary event selection is shown in the left panel of Figure 2 for the May 2018 period, the $\Delta R$ distribution of the reactor on excess is shown in the right panel.
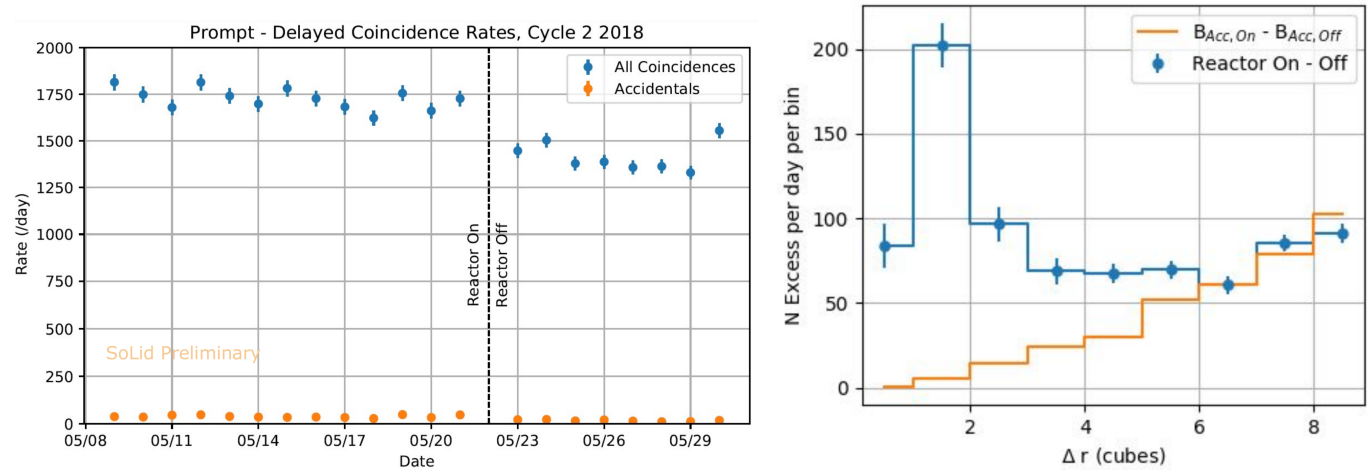

Figure 2: Rate per day of IBD like events in May 2018 (left) and the corresponding $\Delta R$ distribution of the reactor on excess (right). Accidental rates are estimated from an offtime window.

\section{References}

[1] J. Kopp et al. Updated global analysis of neutrino oscillations in the presence of eV-scale sterile neutrinos. JHEP 08 (2018), 010.

[2] C. Giunti et al. Updated Global 3+1 Analysis of Short-BaseLine Neutrino Oscillations. JHEP 06 (2017), 135.

[3] Y. Abreu et al. Optimisation of the scintillation light collextion and uniformity for the SoLid experiment. JINST 13 (2018), P09005.

[4] Scintacor. Neutron screens https://scintacor.com/wpcontent/uploads / 2015/09/Datasheet-Neutron-Screens-High-Res.pdf

[5] Eljen Technology. General Purpose Plastic Scintillator EJ-200, EJ-204, EJ-208, EJ-212. https://el jentechnology.com/images/products/data_sheets/EJ-200_EJ-204_EJ208_EJ-212.pdf

[6] L. Arnold, W. Beaumont, D. Cussans, D. Newbold, N. Ryder and A. Weber, The SoLid antineutrino detector's readout system. JINST 12 (2017), C02012. 